\title{
Experimental study of the anti-cancer mechanism of tanshinone IIA against human breast cancer
}

\author{
QING LU, PURONG ZHANG, XIN ZHANG and JIE CHEN \\ Department of Breast Surgery, West China Hospital, Sichuan University, \\ Chengdu 610041, P.R. China
}

Received June 2, 2009: Accepted July 27, 2009

DOI: $10.3892 /$ ijmm_00000291

\begin{abstract}
Tanshinone IIA is a widely used Chinese herbal medicine isolated from Danshen (Salvia miltiorrhiza). Recent studies indicate that tanshinone IIA may have antiinflammatory and anti-oxidant properties, as well as cytotoxic activities against multiple human cancer cell lines. This study was performed to determine the anticancer activity of tanshinone IIA on human breast cancer cells in vitro and in vivo and to elucidate the underlying mechanism of this activity. Human breast cancer cell lines (estrogen receptor-positive and -negative) were treated with tanshinone IIA and tamoxifen. The inhibitory effects of tanshinone IIA and tamoxifen on breast cancer cell proliferation were examined using MTT assays, BrdU incorporation, immunohistochemistry and flow cytometry. Upon treatment with tanshinone IIA, breast cancer cell proliferation was significantly inhibited in a dose- and time-dependent manner $\left(\mathrm{IC}_{50}=0.25 \mu \mathrm{g} / \mathrm{ml}\right)$ and apoptotic cell populations increased, while tamoxifen inhibited only ER-positive breast cancer cells prominently and had no effect on ER-negative cells. In addition, tamoxifen had significantly weaker inhibitory effect than tanshinone IIA on ER-positive breast cancer cells in vitro and in vivo. Furthermore, tanshinone IIA decreased the expression of P53 and bcl-2, but not of cerbB-2, in estrogen receptorpositive and negative xenografted nude mice. Our findings suggest that tanshinone IIA might have potential anti-cancer activity that is stronger than tamoxifen in both ER-positive and ER-negative breast cancers. This function could be attributed in part to its inhibition of proliferation and apoptosis induction in cancer cells via the downregulation of multiple genes involved in cell cycle regulation, cell proliferation, apoptosis and DNA synthesis.
\end{abstract}

Correspondence to: Dr Qing Lu, Department of Breast Surgery, West China Hospital, Sichuan University, Chengdu 610041, P.R. China

E-mail: lqlq1963@yahoo.cn

Key words: tanshinone IIA, breast cancer, tamoxifen, growth inhibition, apoptosis

\section{Introduction}

Breast carcinoma is a disease with a high incidence among women, especially in Western countries; it is the most common malignant tumor in women (1). Until now, integrated treatment (surgery, chemotherapy, radiotherapy, endocrine therapy) has greatly reduced the mortality of this malignant tumor (2), but patients with estrogen receptor (ER)-negative cancer have a poor prognosis because these patients are not sensitive to endocrine therapy. Thus, additional preventive and therapeutic modalities for both ER-positive and ER-negative patients are required (3).

Danshen (Salvia miltiorrhiza Bunge) has been widely used in the practice of traditional Chinese medicine; diterpene quinone and phenolic acid derivatives, including tanshinone (I, IIA and IIB), cryptotanshinone, isocryptotanshinone, miltirone, tanshinol (I and II) and salviol are the major extractable components of Danshen. Tanshinone IIA, a major extractable component of danshen, has previously been reported to possess anti-oxidative effects and to inhibit the association of lipid peroxidation products with DNA by breaking the chain reactions associated with peroxidation and scavenging lipid-free radicals (4-7). The planar phenanthrene ring of the tanshinones may be essential for their interaction with DNA molecules, whereas the furano-oquinone moiety could be responsible for the production of reactive-free radicals in close vicinity to bases, causing DNA damage and inducing cytotoxicity in different human cancer cell lines (8). Several studies have shown that tanshinone IIA inhibits the proliferation of mouse P388 lymphocytic leukemia cells (9) and human hepatoma cells in vitro (10-13); tanshinone IIA can also induce apoptosis in human hepatocellular carcinoma cells (11-13), human promyelocytic leukemia cells (HL60), and human erythroleukemic cells (K562) (14,15); Finally, tanshinone IIA has been shown to induce differentiation of human leukemia cells (16-18). Recently, tanshinone IIA has been reported to inhibit the proliferation of the ER-positive human breast cancer cells MCF-7 (19) and the ER-negative cells MDA-MB-231 in vitro (20); the associated mechanism, however, is not clearly understood.

In the present study, we examined whether tanshinone IIA has an effect on breast cancer cells in vitro and in vivo using ER-positive MCF-7 and ER-negative MDA-MBA-231 cells. 
We also investigated the mechanisms underlying the effects of the activity of tanshinone IIA on these cells.

\section{Materials and methods}

Cell lines and cultures. Human breast cancer MCF-7 (ERpositive) and MDA-MB-231(ER-negative) cell lines were provided by Shanghai Institute of Cancer Research. Cells were maintained in RPMI-1640 supplemented with $10 \%$ FBS, penicillin $(100 \mathrm{U} / \mathrm{ml})$ and streptomycin $(100 \mathrm{mg} / \mathrm{ml})$ in a humidified atmosphere of $50 \mathrm{mg} / \mathrm{ml} \mathrm{CO}_{2}$ at $37^{\circ} \mathrm{C}$. The study protocol was approved by Sichuan University.

Chemicals and reagents. Tanshinone IIA (Tan IIA) was purchased from the Chinese Institute for Drug and Biological Product Control (concentration $\geq 98 \% /$ HPLC, Beijing, P.R. China). Tamoxifen was purchased from Sigma (St. Louis, MO, USA). Mouse anti-human P53, cerbB-2 and bcl-2 monoclonal antibodies were obtained from Zhongshan Goldenbridge Biotechonology (Beijing, P.R. China). Other special chemicals were purchased from Sigma.

Cytotoxicity assays (MTT assay). The cytotoxicity of tanshinone IIA was determined using the MTT assay. Both cell lines $\left(2 \times 10^{4} /\right.$ well) were plated in $100 \mu \mathrm{l}$ of medium/well in 96-well plates (Costar Corning, Rochester, NY). After incubation overnight, tanshinone IIA and tamoxifen were added at various concentrations $[(0,0.0625,0.125,0.25,0.5$, $1.0 \mu \mathrm{g} / \mathrm{ml})$ and $(0,1,3,5,10$ and $15 \mu \mathrm{M} / \mathrm{l})$, respectively]; four wells were included for each concentration. After treatment with tanshinone IIA for 24, 48, 72, 96 and $120 \mathrm{~h}$, $20 \mu \mathrm{l}$ of $5 \mathrm{mg} / \mathrm{ml}$ MTT (pH 4.7) was added to each well and the mixture was cultivated for another $4 \mathrm{~h}$. The supernatant was then removed, $100 \mu \mathrm{l} /$ well DMSO was added and the samples were shaken for $15 \mathrm{~min}$. The absorbance at $570 \mathrm{~nm}$ was measured with a microplate reader (Bio-Rad, Richmond, CA) using wells without cells as blanks. All experiments were performed in triplicate. The effect of tanshinone IIA and tamoxifen on the proliferation of MCF-7 cells is expressed as the percent cytoviability using the following formula: \% cytoviability $=\mathrm{A}_{570}$ of treated cells $/ \mathrm{A}_{570}$ of control cells $\mathrm{x}$ $100 \%$.

BrdU incorporation in vitro. Cells were seeded onto glass coverslips at an initial density of $2 \times 10^{4} / \mathrm{ml}$ in a 6 -well plate, $3 \mathrm{ml}$ per well. They were allowed to grow for $24 \mathrm{~h}$ and then were treated with $0.25 \mu \mathrm{g} / \mathrm{ml}$ of tanshinone IIA and $10 \mu \mathrm{mol} / \mathrm{l}$ tamoxifen for $48 \mathrm{~h}$. Cells were incubated with BrdU in medium $(20 \mu \mathrm{g} / \mathrm{ml})$ for $24 \mathrm{~h}$. At the appropriate time, cells were fixed in methanol at $-20^{\circ} \mathrm{C}$ for $1-2 \mathrm{~min}$, allowed to air dry and then stored at $-20^{\circ} \mathrm{C}$ until all coverslips were ready for processing. Cells were rehydrated in PBS for $5 \mathrm{~min}$, followed by immersion in $2 \mathrm{~N} \mathrm{HCl}$ for $1 \mathrm{~h}$ at room temperature. Cells were incubated in $0.1 \mathrm{M}$ borate buffer $(\mathrm{pH} 8.5 ; 0.1 \mathrm{M}$ boric acid, $25 \mathrm{mM} \mathrm{Na} \mathrm{B}_{4} \mathrm{O}_{7}$ and $75 \mathrm{mM} \mathrm{NaCl}$ ) twice for $5 \mathrm{~min}$ each, followed by three washes (10 min) in PBS. Next, cells were incubated with BrdU mouse MAb (11B5; Zymed, San Francisco, CA) at a dilution of 1:100 overnight at $4^{\circ} \mathrm{C}$, followed by a biotinylated second antibody for $20 \mathrm{~min}$ and streptavidin/peroxidase for $30 \mathrm{~min}$ at room temperature. The sections were subsequently stained with $0.02 \% 3$, 3'-diaminobenzidine tetrahydrochloride containing $0.005 \% \mathrm{H}_{2} \mathrm{O}_{2}$ in PBS (pH 7.4) and lightly counterstained with hematoxylin. The percentage of BrdU-labeled cells was determined by counting several fields each containing 200 cells (in areas of the slide containing the highest number of labeled cells).

Flow cytometry. Both cell lines were seeded in cell culture bottles at a concentration of $5 \times 10^{5} / \mathrm{ml}$ per bottle. There were three groups in each cell, two bottles per group, resulting in a total of 12 bottles. After incubation for $24 \mathrm{~h}$, the cells were treated with $0.25 \mu \mathrm{g} / \mathrm{ml}$ tanshinone IIA and $10 \mu \mathrm{mol} / 1$ tamoxifen for $72 \mathrm{~h}$. The cells were harvested in $10 \mathrm{ml}$ centrifugal tubes, $800 \mathrm{r} / \mathrm{min}$, centrifuged for $5 \mathrm{~min}$, washed twice using PBS solution and then following another centrifugation $75 \%$ cold ethanol and was added and the cells were fixed for $6 \mathrm{~h}$. The liquid was then removed and the cells washed twice with PBS solution and centrifuged once more, followed by addition of $5 \mu \mathrm{g} / \mathrm{ml}$ PI solution to $1 \mathrm{ml}$. The samples were stained at $4^{\circ} \mathrm{C}$ for $15 \mathrm{~min}$ in the dark and then were detected using a FACS-420 FCM. The apoptosis index was calculated.

Tumor xenograft study in vivo. Thirty-six five-week-old female nude mice (Experimental Animal Center, Sichuan University) with an average weight of $20 \pm 2 \mathrm{~g}$ were randomized into two clusters, each containing 18 nude mice. They were orthotopically xenotransplanted with $0.3 \mathrm{ml}$ human ER-positive breast cancer cells MCF-7 or ER-negative breast cancer cells MDA-MB-231 (the concentration of cancer cell suspensions was $2 \times 10^{7} / \mathrm{ml}$ ). At two weeks, the tumors reached $\sim 40 \mathrm{~mm}^{3}$ in volume and the tumor-bearing mice in each cluster were randomized into three experimental groups. The treatment group $(n=6)$ of each cluster was injected with $30 \mathrm{mg} / \mathrm{kg}$ tanshinone IIA dissolved in $99 \%$ ethanol and $1 \%$ Tween-20 (tanshinone IIA for a final concentration of $10 \mathrm{mg} / \mathrm{ml}$, ethanol concentration of $10 \%$ ) s.c. four times/week for four weeks. The positive control tamoxifen group $(n=6)$ of each cluster was fed tamoxifen (4 mg/kg per day) dissolved in $99 \%$ ethanol and $1 \%$ Tween-20 also for four weeks (tamoxifen at a final concentration of $1 \mathrm{mg} / \mathrm{ml}$, ethanol concentration of $10 \%$ ). The control group $(n=6)$ of each cluster was injected with the same volume of $99 \%$ ethanol and $1 \%$ Tween-20 (ethanol concentration of $10 \%$ ).

Observation and measurement of specimen preparation. The eating, living situation and mass growth of the mice were observed every day. The mice were weighed each week and tumor volumes were assessed by measuring the two perpendicular dimensions using a caliper and the formula $\left(\mathrm{a} \mathrm{x}^{2}\right) / 2$, where $\mathrm{a}$ is the larger and $\mathrm{b}$ is the smaller dimension of the tumor. After four weeks of treatment, mice were euthanized and tumors completely removed in $30 \mathrm{~min}$ and then dissected from their envelopes; tumors were then weighed and the following calculations were performed: tumor volume inhibiting formula $=($ control group tumor volume - experimental group tumor volume/control group tumor volume $\mathrm{x} 100 \%$ and tumor weight inhibiting rate $=$ (control group tumor weight - experimental group tumor weight)/control group tumor weight x $100 \%$. Tumors in each 

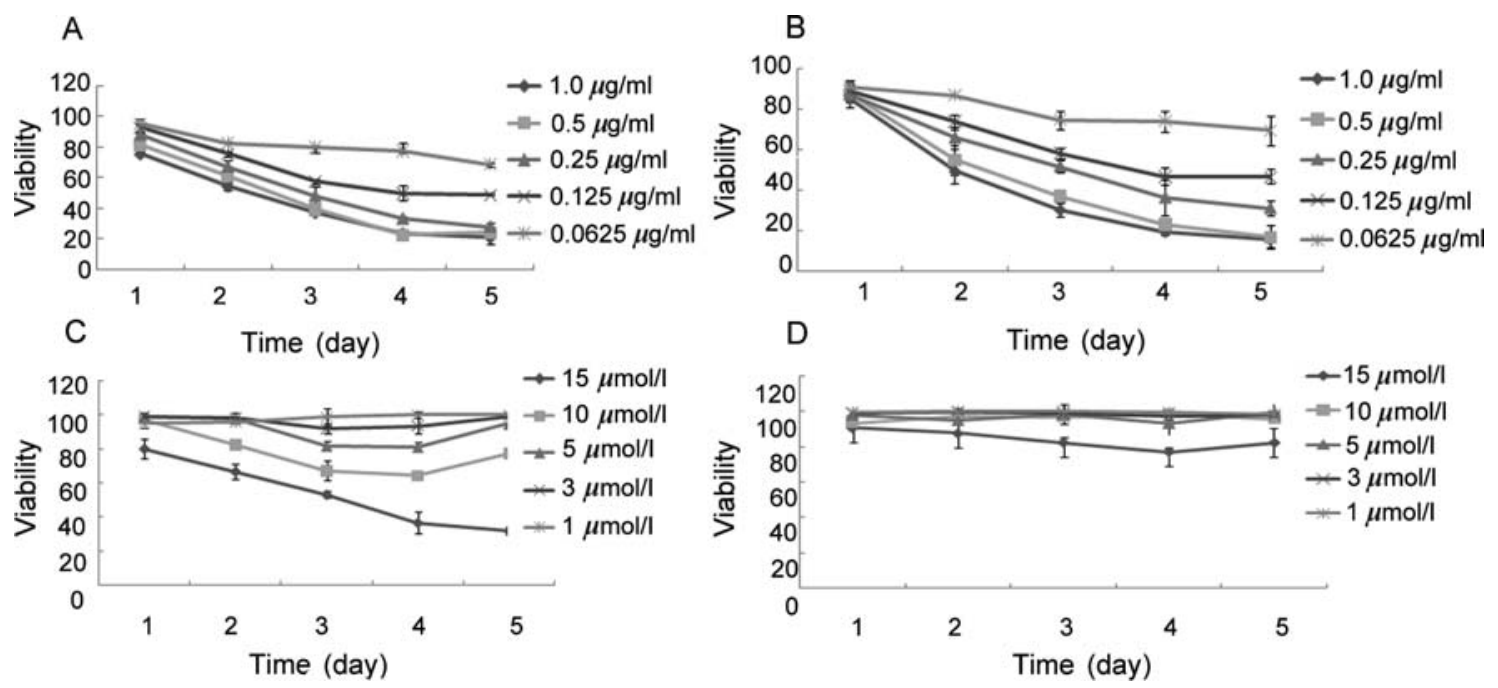

Figure 1. Inhibition of human MCF-7 (A) and MDA-MB-231 (B) breast cancer cell growth by tanshinone IIA. Inhibition of human MCF-7 (C) and MDA-MB-231 (D) breast cancer cell growth by tamoxifen.

group were cut into several pieces, each $\sim 10 \times 10 \times 2 \mathrm{~mm}$ in size, with a portion reserved for flow cytometry detection and other parts paraffin-embedded after fixation in $4 \%$ paraformaldehyde for $24 \mathrm{~h}$.

Immunohistochemical analysis of tumors for P53, cerbB-2, and bcl-2 protein expression. Tumor samples of tanshione IIA, the positive control tamoxifen group and the negative control group from mice were fixed in $4 \%$ paraformaldehyde for $24 \mathrm{~h}$ and processed conventionally. P53, cerbB-2 and bcl-2 protein expression was detected immunohistochemically with the SP kit (Zymed); briefly, tissue sections were deparaffinized and rehydrated in graded alcohol solutions. Antigen retrieval was performed in a $0.1 \mathrm{mM}$ citrate buffer (pH 6.0) by microwave oven heating. Endogenous peroxidase activity was then blocked with $3 \% \mathrm{H}_{2} \mathrm{O}_{2}$; after treatment with normal goat serum, tissue sections were incubated with P53, cerbB-2 and bcl-2 mouse anti-human monoclonal antibodies (Zhongshan Goldenbridge Biotechonology, Beijing, P.R. China) at a dilution of $1: 100$ overnight at $4^{\circ} \mathrm{C}$, followed by biotinylated secondary antibody for $20 \mathrm{~min}$ and streptavidin/peroxidase for $30 \mathrm{~min}$ at room temperature. Subsequently, sections were stained with $0.02 \%$.

3,3'-diaminobenzidine tetrahydrochloride containing $0.005 \% \mathrm{H}_{2} \mathrm{O}_{2}$ in PBS (pH 7.4) and lightly counterstained with hematoxylin. P53-, cerbB-2- and bcl-2-positive cells were identified using the positive intensity (PI) score as follows: no coloring $=0$, light yellow marks $=1$, brown $=2$, dark brown $=3$. Two hundred cells in several fields were assessed to calculate the average PI score for statistical analysis. PI score $=$ total score-positive $/ 200$. The entire process was performed in a blinded fashion.

Statistical analyses. The statistical significance of the difference between the control groups and the tanshinone IIAtreated groups was determined by the Wilcoxon test and compared with the design of various rank sum tests. Diverse rank sum tests for multiple comparisons and Spearman rank correlation analyses was employed as needed. The results were considered significant at $\mathrm{P}<0.05$.

\section{Results}

Growth inhibitory effect of tanshinone IIA on human breast cancer cells. As shown in Fig. 1, we found that tanshione IIA was able to decrease ER-positive human breast cancer cell (MCF-7) and ER-negative human breast cancer cell (MDAMB-231) viability in a dose- and time-dependent manner. The concentrations required to inhibit cell growth by $50 \%$ $\left(\mathrm{IC}_{50}\right)$ for MCF-7 and MDA-MB-231 were $0.25 \mu \mathrm{g} / \mathrm{ml}$. In addition, we examined the effect of tamoxifen on the survival rate, as shown in Fig 2. We found that tamoxifen inhibited MCF-7 cell growth at concentrations ranging from 5-15 $\mu \mathrm{M} / \mathrm{l}$, which was weaker than the tanshinone IIA growth inhibition curve $(\mathrm{P}<0.05)$; the $\mathrm{IC}_{50}$ was $10 \mu \mathrm{M} / 1$ for tanshinone, whereas tamoxifen inhibited MDA-MB-231 cell growth at a concentration of $15 \mu \mathrm{mol} / 1$ (Fig. 2). This finding was consistent with previous studies showing that tamoxifen has no effect on ERnegative breast cancer cells. Compared with the tamoxifen group, tanshione IIA demonstrated greater inhibitory activity on both MCF-7 and MDA-MB-231 ( $\mathrm{P}<0.05)$.

Inhibition of cell proliferation. MCF-7 cells and MDA-MB-231 cells were further cultured in the presence of tanshinone IIA $(0.25 \mu \mathrm{g} / \mathrm{ml})$ and tamoxifen $(10 \mu \mathrm{mol} / \mathrm{l})$. Cell proliferation was quantified by measuring BrdU incorporation in untreated and treated $(0.25 \mu \mathrm{g} / \mathrm{ml})$ breast cancer cells in vitro. As shown in Figs. 3 and 4, BrdU-labeled cells among tanshinone IIAtreated MCF-7 cells were $23.8 \pm 4.3 \%$, lower than the number obtained for untreated controls $(54.8 \pm 2.3 \%)$ and for the tamoxifen group $(40.8 \pm 6.9 \%)$; both differences were statistically significant $(\mathrm{P}<0.0001)$. In the tanshione IIAtreated group, BrdU-labeled MDA-MB-231 cells were $21.1 \pm 2.8 \%$, which was significantly lower than those quantified in the untreated controls $(43.9 \pm 5.1 \%)$ and in the tamoxifen group $(37.9 \pm 3.9 \%)$ (both $\mathrm{P}<0.0001)$.

Induction of apoptosis by tanshinone IIA. To determine whether the growth inhibitory activity of tanshinone IIA was related to induction of apoptosis, we examined the apoptotic 

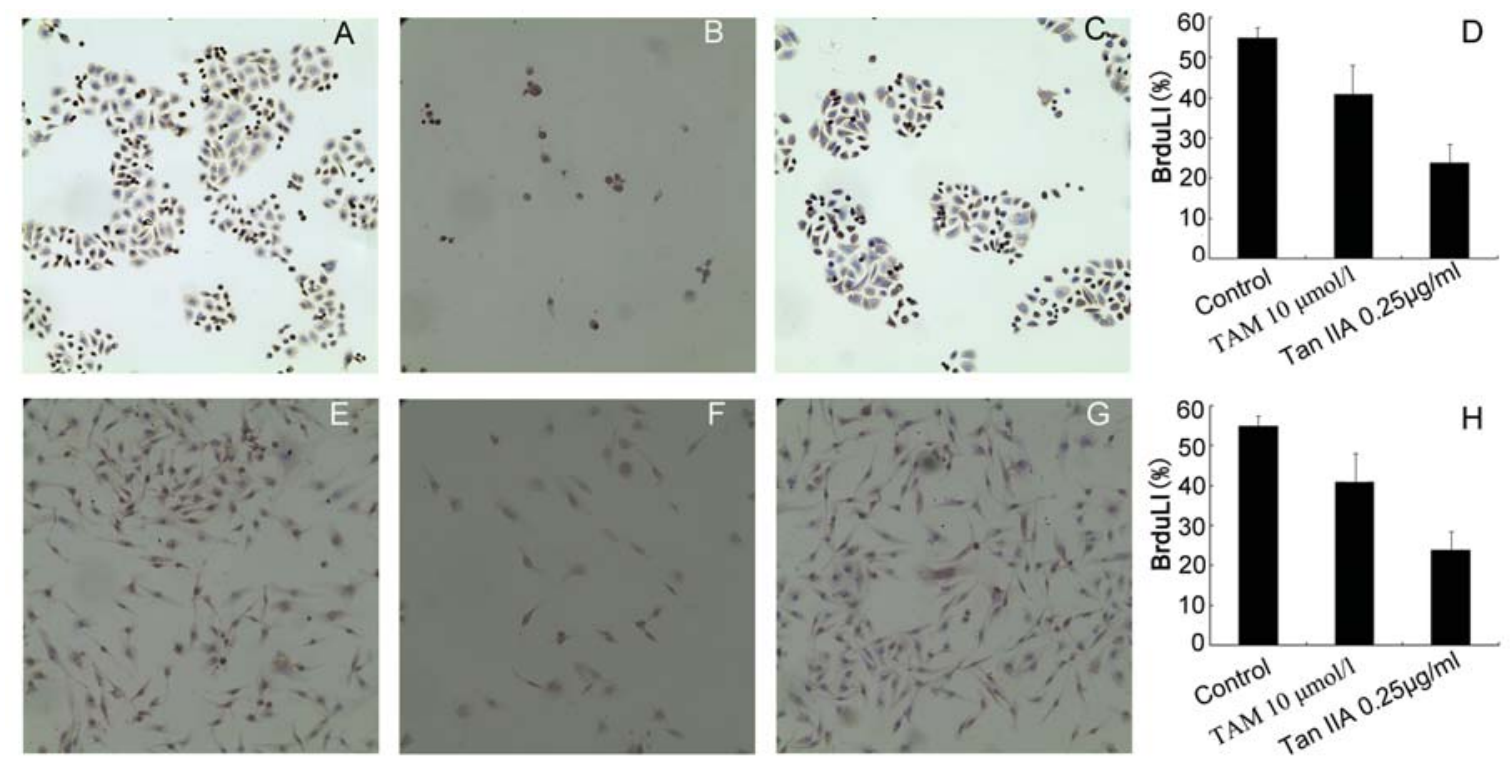

Figure 2. BrdU incorporation in MCF-7 cells in vitro (A) BrdU-labeled cells in the untreated group. (B) Decreased number of BrdU-labeled cells in the tanshione IIA-treated group. (C) Decreased number of BrdU-labeled cells in the tamoxifen group. (D) Histogram showing a significant decrease in BrdU-labeled cells in tanshinone IIA-treated cells compared to the other two groups. BrdU incorporation in MDA-MB-231 cells in vitro (E) BrdU-labeled cells in the untreated group. (F) Decreased number of BrdU-labeled cells in the tanshinone IIA-treated group. (G) The number of Brdu-labeled cells in the tamoxifen group did not change. (H) Histogram showing a significant decrease in BrdU-labeled cells in the tanshinone IIA-treated group.

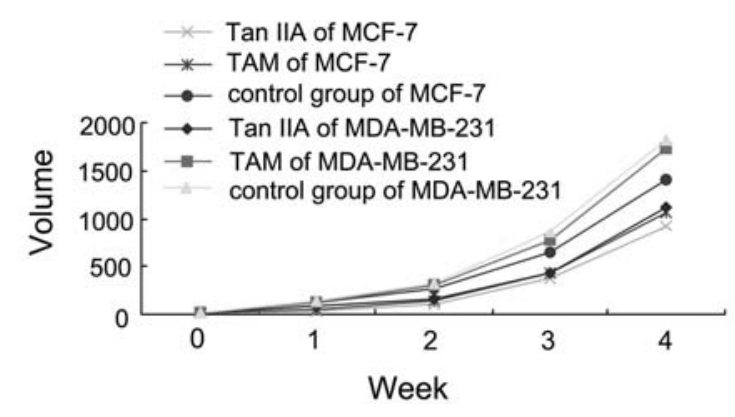

Figure 3. Change in tumor volume according to group $\left(\mathrm{mm}^{3}\right)$.

populations in MCF-7 and MDA-MB-231 cells upon tanshinone IIA and tamoxifen treatment by flow cytometry. In MCF-7 cells, an apoptosis index of $29.9 \pm 5.5 \%$ was observed in the tanshinone IIA-treated groups $(0.25 \mu \mathrm{g} / \mathrm{ml})$, a much higher index than those obtained for the tamoxifen group $(13.4 \pm 1.1 \%)$ and the untreated controls $(6.7 \pm 2.8 \%)$. In MDA-MB-231 cells, an apoptosis index of $23.3 \pm 5.9 \%)$ was observed in the tanshinone IIA-treated groups $(0.25 \mu \mathrm{g} / \mathrm{ml})$, which was much higher than those obtained for the tamoxifen group $(9.5 \pm 6.8 \%)$ and the untreated controls $(6.1 \pm 5.0 \%)$.

Inhibition of tumor growth in human breast cancer cells xenografts in nude mice. To confirm the anti-cancer activity of tanshinone IIA on human breast cancer cells, an in vivo experiment was carried out by xenografting ER-positive human cancer cells MCF-7 and ER-negative human breast cancer cells MDA-MB-231 in an animal model. After tumor inoculation, tanshinone IIA and tamoxifen administration was started (designated as day 0). Tanshinone IIA was administered at $30 \mathrm{mg} \cdot \mathrm{kg}^{-1} \cdot \mathrm{day}^{-1}$ four times/week and tamoxifen was administered at $4 \mathrm{mg} / \mathrm{kg}$ per day for four weeks.
After four weeks of treatment with tanshinone IIA, tumor growth in the MCF-7-treated group was apparently inhibited, as was the case in the tamoxifen group compared with the untreated MCF-7 groups (Figs. 3 and 4). The tumor volume inhibitory rate of tanshinone IIA was $33.64 \%$ and the tumor weight inhibitory rate was $32.24 \%$ (compared with the untreated group: $\mathrm{P}=0.007$ and $\mathrm{P}=0.021$, respectively). The tumor volume inhibitory rate of the tamoxifen group was $24.29 \%$ and the tumor weight inhibitory rate was $26.34 \%$ (compared with the untreated group: $\mathrm{P}=0.023$ and $\mathrm{P}=0.011$, respectively). Tanshione IIA had greater inhibitory activity against MCF-7 cell tumors in nude mice than did tamoxifen $(\mathrm{P}=0.015$ and $\mathrm{P}=0.025$, respectively). The tanshinone IIAtreated MDA-MB-231 group of nude mice displayed slow tumor growth, and the tumor volume inhibitory rate was $38.43 \%$; the tumor weight inhibitory rate was $39.82 \%$ (Figs. 3 and 4), which is significantly more inhibition than in the tamoxifen-treated MDA-MB-231 group. These results suggest that tanshinone IIA is an efficient inhibitor of the growth of human breast cancer cells xenografted into nude mice compared with tamoxifen injections, with inhibition of tumor growth and that tanshinone IIA exhibits anti-tumor activity in vivo. None of the mice died during the experiments.

Cell apoptosis in vivo. The cell apoptosis-inducing activity of tanshinone IIA against human breast cancer cells was also confirmed in an in vivo experiment. In MCF-7-xenografted nude mice, apoptosis indices of $48.31 \pm 5.84 \%$ and $42.95 \pm 4.84 \%$ were observed in the tanshinone IIA- and tamoxifen-treated groups, respectively, both of which were much higher than that obtained for control mice $(29.51 \pm 10.08 \%)$; $(\mathrm{P}=0.004)$. In MDA-MB-231-xenografted nude mice, an apoptosis index of $50.25 \pm 5.03 \%$ was observed in the tanshinone IIA-treated groups, which was much higher than those obtained for the tamoxifen group $(37.88 \pm 3.14 \%)$ and the control mice 

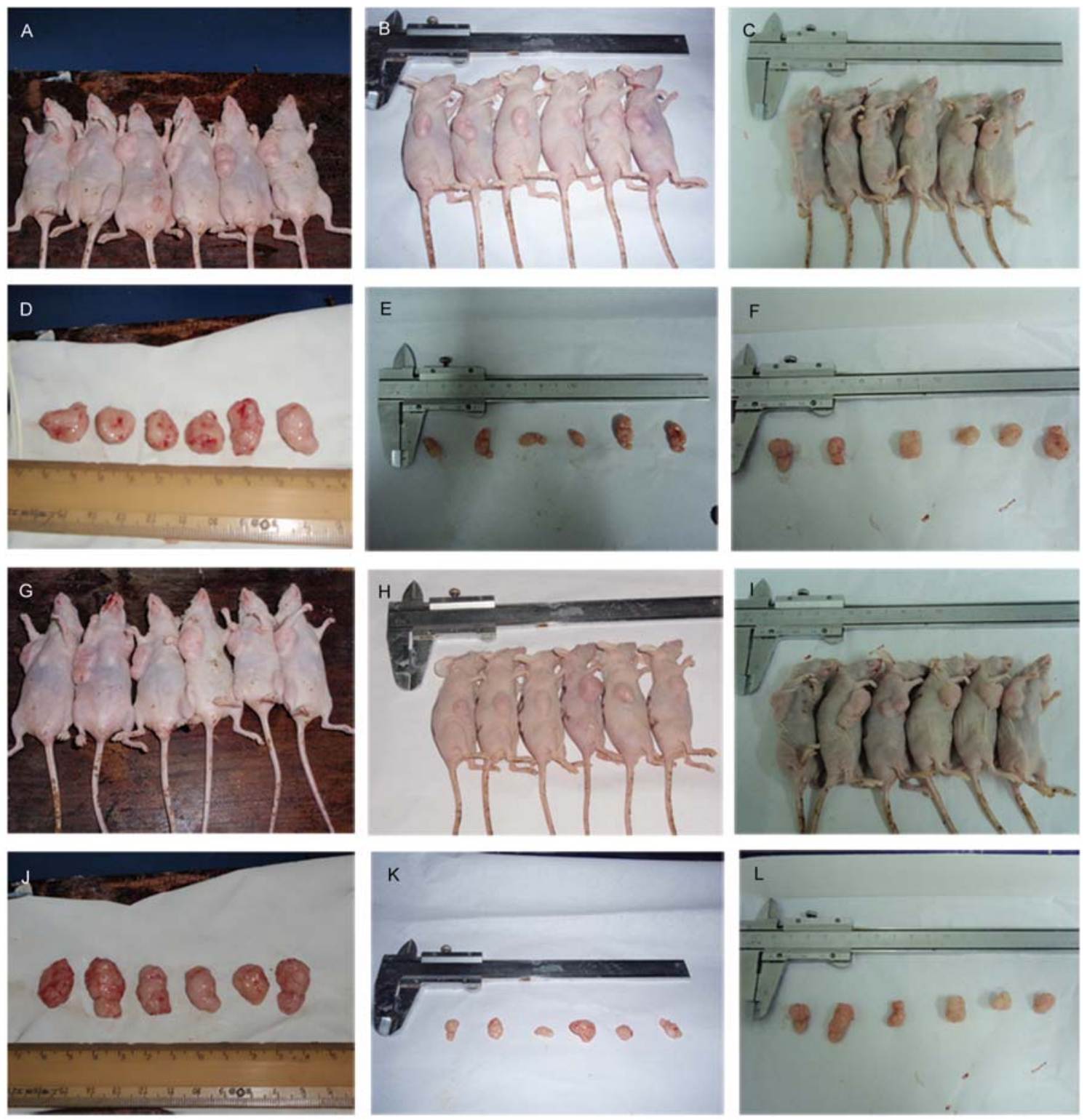

Figure 4. MCF-7 nude mice with tumors (A) control group; (B) Tan IIA group; (C) TAM group. MCF-7 tumors (D) control group; (E) Tan IIA group; (F) TAM group. MDA-MB-231 nude mice with tumors (G) control group; (H) Tan IIA group; (I) TAM group. MDA-MB-231 tumors (J) control group; (K) Tan IIA group; (L) TAM group.

$(35.35 \pm 3.46 \%)$. The apoptosis index of the MDA-MB-231 cells in response to tanshinone IIA treatment was $50.25 \pm 5.03 \%$ and the indices obtained for the control and tamoxifen groups were $35.35 \pm 3.46 \%$ and $37.88 \pm 3.14 \%$, respectively; the treated group displayed a significantly higher value than the other groups $(\mathrm{P}=0.004$ and $\mathrm{P}=0.004$, respectively).

P53, cerbB-2 and bcl-2 protein expression in tumors. Previous studies have shown that P53, cerbB-2 and bcl-2 play pivotal roles in cell growth and apoptosis. We showed that P53 selectively labeled the nuclei, bcl-2 labeled the cytoplasm and cerbB-2 labeled the cell membrane. These positions had a morphology consistent with apoptosis and cell growth (Figs. 5-7). In the MCF-7 nude model, the positive intensity (PI) score of P53 and bcl-2 expression of the tanshinone IIAtreated group were both decreased compared with the control mice $(\mathrm{P}=0.031$ and 0.021$)$; there were no significant differences of cerbB-2 between the two groups $(\mathrm{P}=0.33)$; In the MDA-
MB-231 model, the expression of P53 and bcl-2 was lower than in the control mice $(\mathrm{P}=0.039$ and 0.0031$)$, but no significant difference in the expression of cerbB-2 was found between the two groups $(\mathrm{P}=0.19)$; PI score data are shown in Table I.

\section{Discussion}

It has been reported that tanshinone IIA has anti-oxidant properties and cytotoxic activities against multiple human cancer cell lines (8) that inhibit the proliferation of mouse P388 lymphocytic leukemia cells (9) and human hepatoma cells (10-13). Other studies have shown that tanshinone IIA induces apoptosis of human hepatocellular carcinoma cells (11-13), human promyelocytic leukemia cells (HL60) and human erythroleukemia cells (K562) $(14,15)$ and induces differentiation of human leukemia cells (16-18). Wang et al reported that tanshione IIA can inhibit proliferation of ER- 

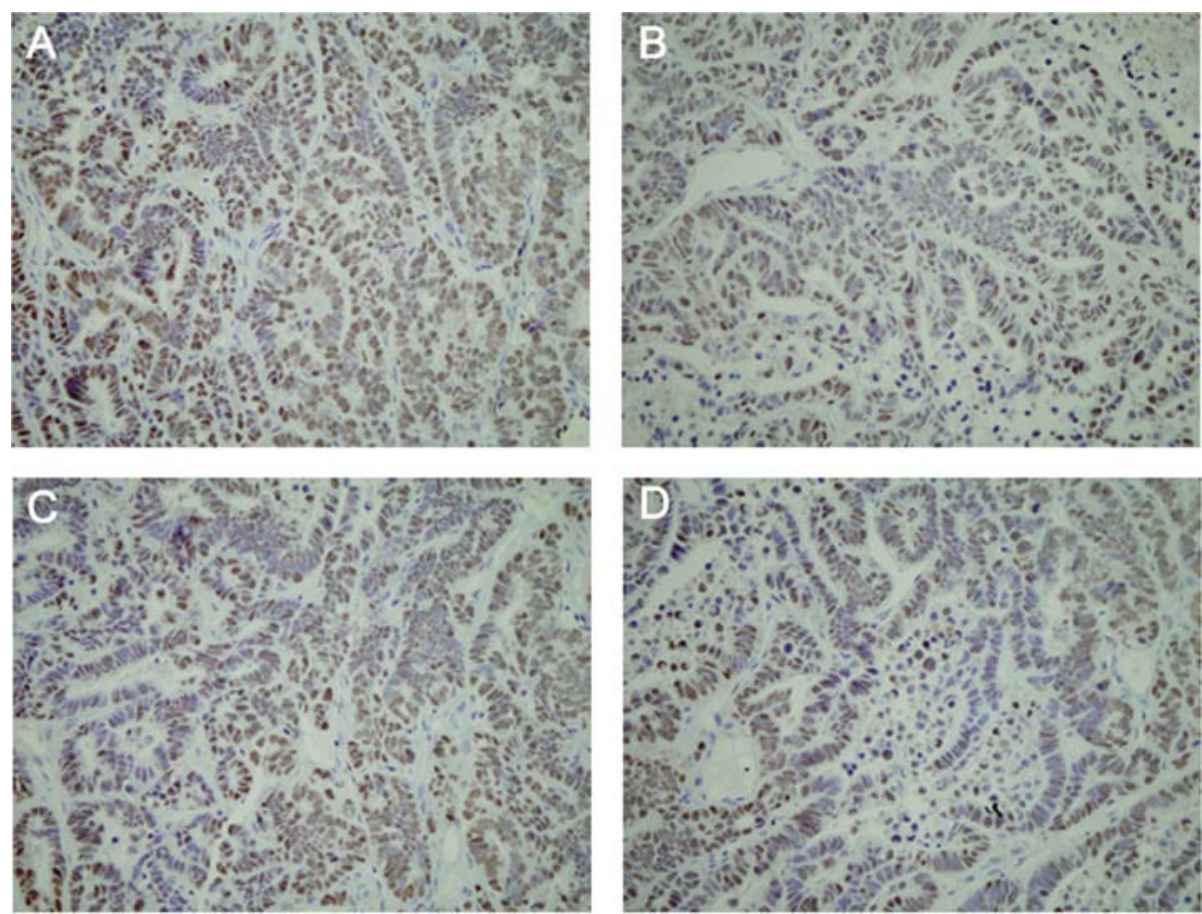

Figure 5. P53 expression in MCF-7 cells (SP x 400) (A) control group; (B) Tan IIA group. P53 expression in MDA-MB-231 cells (SP x 400) (C) control group; (D) Tan IIA group.
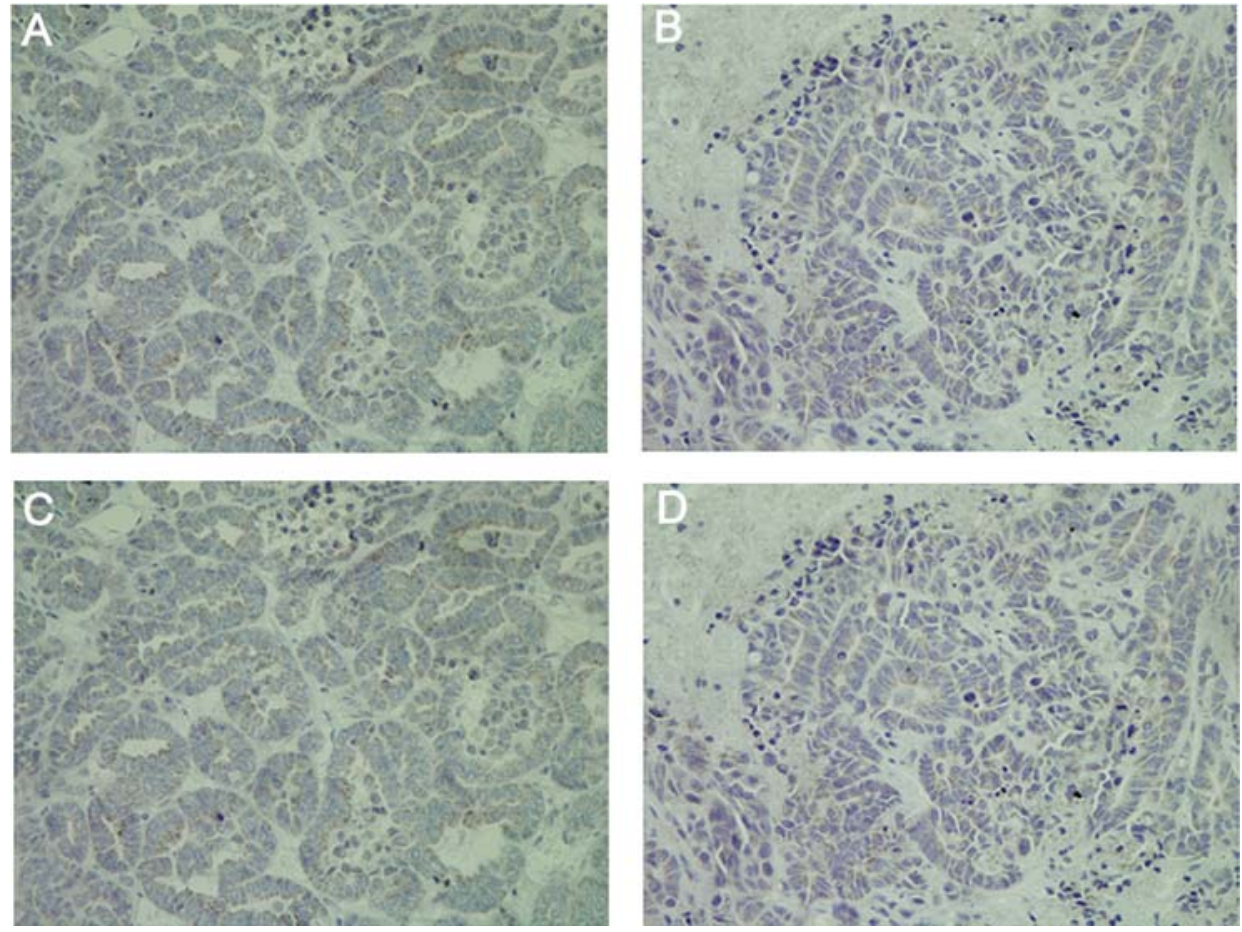

Figure 6. Bcl-2 expression in MCF-7 cells (SP x 400) (A) control group; (B) Tan IIA group. Bcl-2 expression in MDA-MB-231 cells (SP x 400) (C) control group; (D) Tan IIA group.

positive human breast cells in vitro and also inhibit the growth of human ER-negative breast IDC (infiltrating ductal carcinoma) in vivo (19). Recently, Jing et al found that tanshione IIA also has an inhibitory effect on ER-negative human breast cancer cells in vitro (20). However, to our knowledge, no systematic studies investigating the effect of tanshinone IIA on ER-positive human breast cancer cells in vivo or on the differential inhibitory effect on ER-positive and ER-negative human breast cancer cells in vivo have been reported. Tamoxifen has been one of the first-line drugs integral to endocrine therapy of ER-positive breast cancer for many years (21); its pharmacological mechanism can be 

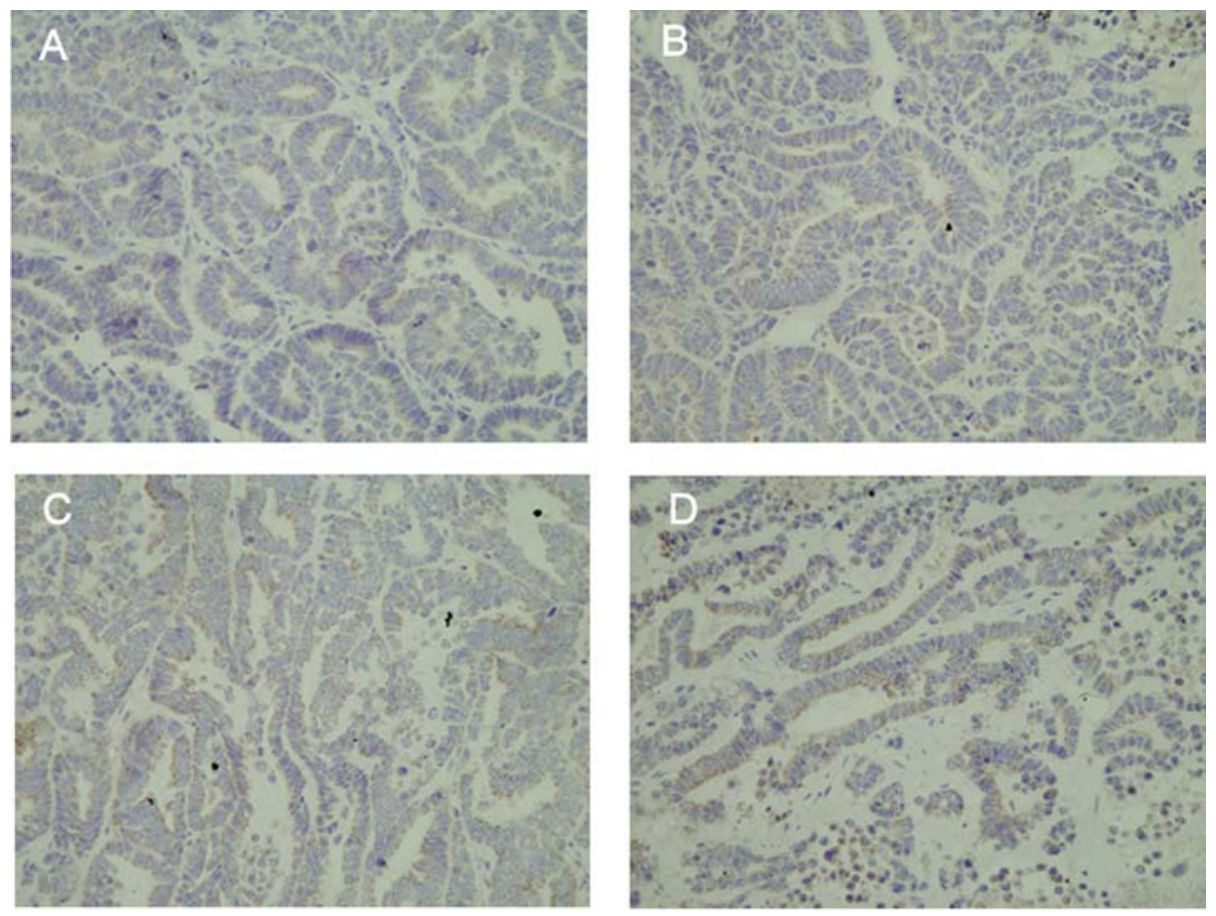

Figure 7. CerbB-2 expression in MCF-7 cells (SP x 400) (A) control group; (B) Tan IIA group. CerbB-2 expression in MDA-MB-231 cells (SP x 400) (C) control group; (D) Tan IIA group.

Table I. Positive intensity (PI) score comparison of protein expression in tumors dealt with by tanshinone IIA after 4 weeks.

\begin{tabular}{lcccccccc}
\hline & \multicolumn{2}{c}{ P53 PI score } & & \multicolumn{2}{c}{ CerbB-2 PI score } & & \multicolumn{2}{c}{ Bcl-2 PI score } \\
\cline { 2 - 3 } & MCF-7 & MDA-MB-231 & MCF-7 & MDA-MB-23 & & MCF-7 & MDA-MB-231 \\
\hline Control G & $1.175 \pm 0.056$ & $1.128 \pm 0.068$ & & $0.551 \pm 0.038$ & $0.982 \pm 0.045$ & & $0.277 \pm 0.037$ & $0.615 \pm 0.078$ \\
Tan IIA G & $0.595 \pm 0.054$ & $0.659 \pm 0.044$ & & $0.532 \pm 0.015$ & $0.944 \pm 0.068$ & & $0.175 \pm 0.086$ & $0.468 \pm 0.066$ \\
P-value & 0.031 & 0.039 & & 0.33 & 0.19 & & 0.021 & 0.0031 \\
\hline
\end{tabular}

interpreted as competition with estrogen for the ER binding site to inhibit the effects of estrogen (22). Our study confirms that tanshinone IIA has strong dose-dependent and timedependent anti-cancer activity in both ER-positive and ERnegative human breast cancer cells $\left(\mathrm{IC}_{50}=0.25 \mu \mathrm{g} / \mathrm{ml}\right)$ in vitro that is stronger than that of tamoxifen. Cultured cancer cells are valuable reagents for the rapid screening of potential anticancer agents, as well as for elucidation of the mechanism responsible for their activity. Prior to clinical trials, however, it is essential that the in vivo efficacy of potential anti-cancer agents be determined in a suitable animal model (23). It is known that approximately one-third of breast cancers are ERnegative, carrying a worse prognosis than the ER-positive ones (3). Therefore, it is important to discover new agents that are effective in the context of both ER-positive and ER-negative breast cancers. Our results not only demonstrate that tanshinone IIA has a powerful inhibitory effect on the proliferation of both ER-positive and ER-negative human breast cells in vitro, but also confirm its significant inhibitory effects on the growth of human ER-positive (MCF-7) and ER-negative (MDA-MB231) breast cancer cells in vivo without any untoward toxicity $(24,25)$. Our findings suggest that tanshinone IIA might have potential anti-cancer activity against human breast cancers, while no difference in the anti-tumor activity of tanshinone IIA in either the presence or absence of estrogen was observed and its action was independent of an estrogen-regulatory mechanism.

The anti-cancer activity of tanshinone IIA might result, at least in part, from its inhibition of DNA synthesis, inhibition of proliferation and induction of apoptosis in cancer cells. Inhibition of DNA synthesis and proliferation of cancer cells was verified by the ability of tanshinone IIA to reduce BrdU incorporation into cancer cells, correlating with decreased cell proliferation (26). After treatment with tanshinone IIA, BrdU-labeled cells were significantly reduced in both ERpositive and ER-negative cells $(\mathrm{P}<0.05)$, indicating that tanshinone IIA inhibited the proliferation of cancer cells. We also showed that tanshinone IIA-induced apoptosis is associated with decreased expression of P53 and bcl-2 proteins in ER-positive and ER-negative tumors in vivo.

In summary, the potential anti-cancer activity of tanshinone IIA against human breast cancer cells was investigated. Tanshinone IIA exhibited a strong inhibitory effect on the proliferation of ER-positive and ER-negative breast cancer 
cells in vitro and also inhibited growth of ER-positive and ER-negative breast cancer cells in vivo by down-regulating the apoptosis-related genes P53 and bcl-2. It also showed a stronger anti-cancer ability than tamoxifen on ER-positive cancer cells both in vitro and in vivo.

\section{Acknowledgements}

This study was supported by Applied Basic Research Programs of Science and Technology Commission of Sichuan Province (0040205301143).

\section{References}

1. Chow LW and Loo WT: The differential effects of cyclophosphamide, epirubicin and 5-fluorouracil on apoptotic marker (CPP-32), pro-apoptotic protein (p21(WAF-1)) and anti-apoptotic protein (bcl-2) in breast cancer cells. Breast Cancer Res Treat 80: 239-244, 2003.

2. Mukherjee AK, Basu S, Sarkar N and Ghosh AC: Advances in cancer therapy with plant based natural products. Curr Med Chem 8: 1467-1486, 2001.

3. Swami S, Raghavachari N, Muller UR, Bao YP and Feldman D: Vitamin D growth inhibition of breast cancer cells: gene expression patterns assessed by cDNA microarray. Breast Cancer Res Treat 80: 49-62, 2003.

4. Cao EH, Liu XQ, Wang JJ and Xu NF: Effect of natural antioxidant tanshinone II-A on DNA damage by lipid peroxidation in liver cells. Free Radic Biol Med 20: 801-806, 1996.

5. Wang AM, Sha SH, Lesniak W and Schacht J: Tanshinone (Salviae miltiorrhizae extract) preparations attenuate aminoglycoside-induced free radical formation in vitro and ototoxicity in vivo. Antimicrob Agents Chemother 47: 1836-1841, 2003.

6. Niu XL, Ichimori K, Yang X, Hirota Y, Hoshiai K, Li M and Nakazawa H: Tanshinone II-A inhibits low density lipoprotein oxidation in vitro. Free Radic Res 33: 305-312, 2000.

7. Zhou GY, Zhao BL, Hou JW, Ma GE and Xin WJ: Protective effects of sodium tanshinone IIA sulphonate against adriamycininduced lipid peroxidation in mice hearts in vivo and in vitro. Pharmacol Res 40: 487-491, 1999.

8. Wu WL, Chang WL and Chen CF: Cytotoxic activities of tanshinones against human carcinoma cell lines. Am J Chin Med 19: 207-216, 1991.

9. Mosaddik MA: In vitro cytotoxicity of tanshinones isolated from Salvia miltiorrhiza Bunge against P388 lymphocytic leukemia cells. Phytomedicine 10: 682-685, 2003.

10. Wang X, Yuan S, Huang R and Song Y: An observation of the effect of tanshinone on cancer cell proliferation by Brdu and PCNA labeling. Hua Xi Yi Ke Da Xue Xue Bao 27: 388-391, 1996.

11. Tang Z, Tang Y and Fu L: Growth inhibition and apoptosis induction in human hepatoma cells by tanshinone II A. J Huazhong Univ Sci Technolog Med Sci 23: 166-168, 172, 2003.
12. Tang ZZ, Tang Y and Fu LB: Effect of tanshinone IIA on the growth behavior of human hepatoma cell line BEL-7402 in vitro and its mechanism. Di Yi Jun Yi Da Xue Xue Bao 23: 595-597, 601, 2003.

13. Yuan SL, Wei YQ, Wang XJ, Xiao F, Li SF and Zhang J: Growth inhibition and apoptosis induction of tanshinone II-A on human hepatocellular carcinoma cells. World J Gastroenterol 10: 2024-2028, 2004.

14. Sung HJ, Choi SM, Yoon Y and An KS: Tanshinone IIA, an ingredient of Salvia miltiorrhiza BUNGE, induces apoptosis in human leukemia cell lines through the activation of caspase-3. Exp Mol Med 31: 174-178, 1999.

15. Yoon Y, Kim YO, Jeon WK, Park HJ and Sung HJ: Tanshinone IIA isolated from Salvia miltiorrhiza BUNGE induced apoptosis in HL60 human premyelocytic leukemia cell line. J Ethnopharmacol 68: 121-127, 1999.

16. Liang Y, Yang Y, Yuan S, et al: Terminal differentiation of human acute promyelocytic leukemia (APL) cells induced by Tanshinone II A in primary culture. Hua Xi Yi Ke Da Xue Xue Bao 31: 207-210, 2000.

17. Wu Y, Yang Y, Meng W, Li Y, Jia Y and Liu T: Study on the differentiation of K562 cell-line induced by Tanshinone II A. Hua Xi Yi Ke Da Xue Xue Bao 33: 80-83, 2002.

18. Wang ZY: Ham-Wasserman lecture: treatment of acute leukemia by inducing differentiation and apoptosis. Hematology Am Soc Hematol Educ Program pp1-13, 2003.

19. Wang X, Wei Y, Yuan S, Liu G, Lu Y, Zhang J and Wang W: Potential anticancer activity of tanshinone IIA against human breast cancer. Int J Cancer 116: 799-807, 2005.

20. Jing J, Zheng H, Wang J, et al: Growth inhibition and multidrug resistance-reversing effect of tanshinone I A on human breast cancer cell with estrogen receptor negative. Sichuan Da Xue Xue Bao Yi Xue Ban 38: 391-395, 2007.

21. Tamoxifen for early breast cancer: an overview of the randomised trials. Early Breast Cancer Trialists' Collaborative Group. Lancet 351: 1451-1467, 1998.

22. Wong ZW and Ellis MJ: First-line endocrine treatment of breast cancer: aromatase inhibitor or antioestrogen? Br J Cancer 90: 20-25, 2004.

23. Singh AV, Xiao D, Lew KL, Dhir R and Singh SV: Sulforaphane induces caspase-mediated apoptosis in cultured PC-3 human prostate cancer cells and retards growth of PC-3 xenografts in vivo. Carcinogenesis 25: 83-90, 2004.

24. Liu Y and Wang X: Protective effects of tanshinone IIA on injured primary cultured rat hepatocytes induced by CCl4. Zhong Yao Cai 26: 415-417, 2003.

25. Liu Y, Chen $\mathrm{H}$ and Jiang Y: Protective effect of tanshinone IIA on acute hepatic injury in mice. Zhong Yao Cai 24: 588-589, 2001.

26. Milosevic J, Kanazir S, Medic-Mijacevic L, et al: Sulfinosineinduced cell growth inhibition and apoptosis in human lung carcinomas in vitro. Invest New Drugs 20: 229-240, 2002. 